Delft University of Technology

\title{
Laplacian eigenmaps for multimodal groupwise image registration
}

Polfliet, Mathias; Klein, Stefan; Niessen, Wiro; Vandemeulebroucke, Jef

DOI

$10.1117 / 12.2248719$

Publication date

2017

Document Version

Final published version

Published in

Medical Imaging 2017: Image Processing

\section{Citation (APA)}

Polfliet, M., Klein, S., Niessen, W. J., \& Vandemeulebroucke, J. (2017). Laplacian eigenmaps for multimodal groupwise image registration. In M. A. Styner, \& E. D. Angelini (Eds.), Medical Imaging 2017: Image Processing [101331N] (Proceedings of SPIE; Vol. 10133). Bellingham, WA, USA: SPIE. https://doi.org/10.1117/12.2248719

\section{Important note}

To cite this publication, please use the final published version (if applicable).

Please check the document version above.

\section{Copyright}

Other than for strictly personal use, it is not permitted to download, forward or distribute the text or part of it, without the consent of the author(s) and/or copyright holder(s), unless the work is under an open content license such as Creative Commons.

Takedown policy

Please contact us and provide details if you believe this document breaches copyrights.

We will remove access to the work immediately and investigate your claim. 


\section{Laplacian eigenmaps for multimodal groupwise image registration}

Mathias Polfliet, Stefan Klein, Wiro J. Niessen, Jef Vandemeulebroucke

Mathias Polfliet, Stefan Klein, Wiro J. Niessen, Jef Vandemeulebroucke, "Laplacian eigenmaps for multimodal groupwise image registration," Proc. SPIE 10133, Medical Imaging 2017: Image Processing, 101331N (24 February 2017); doi: 10.1117/12.2248719

SPIE. Event: SPIE Medical Imaging, 2017, Orlando, Florida, United States 


\title{
Laplacian eigenmaps for multimodal groupwise image registration
}

\author{
Mathias Polfliet ${ }^{\mathrm{a}, \mathrm{b}, \mathrm{c}}$, Stefan Klein ${ }^{\mathrm{c}}$, Wiro J. Niessen ${ }^{\mathrm{c}, \mathrm{d}}$, and Jef Vandemeulebroucke ${ }^{\mathrm{a}, \mathrm{b}}$ \\ ${ }^{a}$ Vrije Universiteit Brussel (VUB), Department of Electronics and Informatics (ETRO), \\ Pleinlaan 2, 1050 Brussels, Belgium \\ ${ }^{b}$ imec, Kapeldreef 75, B-3001 Leuven, Belgium \\ ${ }^{c}$ Biomedical Imaging Group Rotterdam, Depts. of Medical Informatics and Radiology, \\ Erasmus MC, the Netherlands \\ ${ }^{\mathrm{d}}$ Imaging Science and Technology, Department of Applied Sciences, Delft University of \\ Technology, Delft, the Netherlands
}

\begin{abstract}
Multimodal groupwise registration has been of growing interest to the image processing community due to developments in scanner technologies (e.g. multiparametric MRI, DCE-CT or PET-MR) that increased both the number of modalities and number of images under consideration. In this work a novel methodology is presented for multimodal groupwise registration that is based on Laplacian eigenmaps, a nonlinear dimensionality reduction technique. Compared to recently proposed dissimilarity metrics based on principal component analysis, the proposed metric should enable a better capture of the intensity relationships between different images in the group. The metric is constructed to be the second smallest eigenvalue from the eigenvector problem defined in Laplacian eigenmaps. The method was validated in three distinct experiments: a non-linear synthetic registration experiment, the registration of quantitative MRI data of the carotid artery, and the registration of multimodal data of the brain (RIRE). The results show increased accuracy and robustness compared to other state-of-the-art groupwise registration methodologies.
\end{abstract}

Keywords: Groupwise registration, multimodal registration, Laplacian eigenmaps, algebraic connectivity

\section{INTRODUCTION}

In radiology, advancements in scanner technologies are leading to an increasing amount of multi-image acquisitions, such as PET-MR, multiparametric MRI and DCE-CT, in which multiple images are acquired during the same session. Patient or organ motion may require the frames to be registered prior to visual inspection or further processing, which may be challenging in the case of multimodal images, or cumbersome in case of large amount of images.

Mutual information ${ }^{1,2}$ is often used in multimodal pairwise registration. Registering a group of multimodal images can be performed by choosing a single image as a reference and registering all other images to this single reference image. Alternatively, one could register a group of images in a groupwise manner where all transformations are estimated at the same time. The advantages of using a groupwise approach are twofold. First, no choice of reference image is made and its bias is avoided. Secondly, all information present in the group of images is considered simultaneously. Examining all images simultaneously could lead to a better tissue differentiation and a better registration result compared to pairwise registration where image information of only two image is taken into account. However, the extension of mutual information to groupwise registration is not straightforward with sparsity in the joint probability density function being the limiting factor. Multimodal groupwise registration has been an active area of research since current methodologies remain application-specific. ${ }^{3-6}$

In this work we propose an alternative dissimilarity metric to perform multimodal groupwise registration based on Laplacian eigenmaps, ${ }^{7}$ a non-linear dimensionality reduction technique. Laplacian eigenmaps have

Further author information: (Send correspondence to M. Polfliet)

M. Polfliet: E-mail: mpolflie@etrovub.be

Medical Imaging 2017: Image Processing, edited by Martin A. Styner, Elsa D. Angelini, Proc. of SPIE Vol. 10133, 101331N · C 2017 SPIE · CCC code: 1605-7422/17/\$18 · doi: 10.1117/12.2248719 
been employed in previous work as a means to perform a multimodal registration through the extraction of structural features. ${ }^{8-11}$ However, our application of Laplacian eigenmaps is based on a different approach, where we consider the temporal (in the case of motion analysis), longitudinal (when monitoring changes in anatomy over time), or cross-sectional (in the case of population analysis) dimension as the dimension to be reduced. The performance of the proposed dissimilarity metric is evaluated on three distinct datasets and compared to other state-of-the-art registration methodologies.

\section{METHODS AND MATERIALS}

\subsection{Groupwise Registration}

In groupwise registration a group of $n$ images is considered for which the transformations to a common reference frame are unknown. The following optimization problem can be considered to determine these transformations:

$$
\hat{\boldsymbol{\mu}}=\arg \min _{\boldsymbol{\mu}} \mathcal{C}\left(I_{1} \circ \mathcal{T}_{\boldsymbol{\mu}_{1}}, \ldots, I_{n} \circ \mathcal{T}_{\boldsymbol{\mu}_{n}}\right),
$$

where $\mathcal{T}_{\boldsymbol{\mu}_{i}}$ is the transformation, parametrized by $\boldsymbol{\mu}_{i}$, that maps the coordinates from the common reference domain to the domain of the $i^{t h}$ image, and $I_{i}$ is the intensity function of the $i^{t h}$ image. $\boldsymbol{\mu}$ is defined to be the vector formed by the concatenation of all separate transformation parameters, $\boldsymbol{\mu}_{i}$ and $\mathcal{C}$ the cost function which is typically defined as the weighted sum of a dissimilarity metric, $\mathcal{D}$, and a regularizer, $\mathcal{R}$.

Furthermore, let us represent each image as a column vector containing its intensities at a fixed number $s$, of sampled coordinates. A matrix representing all images in the group can then be constructed by joining all column representations into a single $s \times n$ matrix $\boldsymbol{M}$. We will denote the column vector $\boldsymbol{m}_{i}$ as the $i^{\text {th }}$ column and the row vector $\boldsymbol{m}^{j}$ as the $j^{\text {th }}$ row of the matrix $\boldsymbol{M}$.

\subsection{PCA-based metrics}

Huizinga et al. proposed two dissimilarity metrics based on PCA for groupwise registration in quantitative MRI data $^{5}$

$$
\begin{gathered}
\mathcal{D}_{P C A}\left(I_{1} \circ \mathcal{T}_{\boldsymbol{\mu}_{1}}, \ldots, I_{n} \circ \mathcal{T}_{\boldsymbol{\mu}_{n}}\right)=n-\sum_{i=1}^{g} \lambda_{i} \\
\mathcal{D}_{P C A 2}\left(I_{1} \circ \mathcal{T}_{\boldsymbol{\mu}_{1}}, \ldots, I_{n} \circ \mathcal{T}_{\boldsymbol{\mu}_{n}}\right)=\sum_{i=1}^{n} i \lambda_{i}
\end{gathered}
$$

where $g$ is a user set parameter and $\lambda_{i}$ is the $i^{t h}$ eigenvalue of the correlation matrix, $\boldsymbol{C}$, of $\boldsymbol{M}$ :

$$
\boldsymbol{C}=\frac{1}{s-1} \boldsymbol{\Sigma}^{-1}(\boldsymbol{M}-\overline{\boldsymbol{M}})^{T}(\boldsymbol{M}-\overline{\boldsymbol{M}}) \boldsymbol{\Sigma}^{-1} .
$$

Herein, $\boldsymbol{\Sigma}$ is a diagonal matrix with its elements equal to the standard deviations of the columns of the data matrix $\boldsymbol{M}$, and $\overline{\boldsymbol{M}}$ is the matrix with its elements equal to the column-wise averages of $\boldsymbol{M}$. The different modalities are thus interpreted as different features, and each sampled coordinate is considered as a separate observation.

In the work by Huizinga et al., the underlying assumption for the PCA-based metrics is that of a linear relation between the intensities of the respective images, or that this relation can be adequately approximated by one. At optimal alignment, the data should therefor lie on (or close to) a hyperplane in the joint intensity space. When moving away from this optimal alignment, this low-dimensional model is disrupted and less variance can be explained by the first eigenvalues of the correlation matrix. While PCA is perfectly suited for modalities with linear relationships, only few modalities truly adhere to such a model. Consequently, PCA-based metrics ignore any non-linearities which could have aided in the registration. Additionaly, more complex non-linear (or stochastical) relationships amongst the images cannot be approximated by a low-dimensional model. 


\subsection{Laplacian Eigenmaps}

With PCA being a linear dimensionality reduction technique, several methods exist for non-linear dimensionality reduction (also known as manifold learning), ${ }^{12}$ one of which is Laplacian eigenmaps (LE). ${ }^{7}$ The LE algorithm is subdivided in three steps. First, an undirected graph is constructed in which every row $\boldsymbol{m}^{j}$ of the data matrix $\boldsymbol{M}$ is a node in the graph. Each node is connected with an edge to its $k$ nearest neighbours. Secondly, the $s \times s$ weight matrix, $\boldsymbol{W}$, is constructed as

$$
W_{a b}= \begin{cases}0 & \text { if } a \text { and } b \text { are not connected } \\ e^{-\frac{\left\|m^{a}-m^{b}\right\|^{2}}{t}} & \text { if } a \text { and } b \text { are connected }\end{cases}
$$

and where $t$ is a user set constant. Finally, the manifold is embedded into a lower dimension by optimally preserving the locality, i.e. the distance between the nodes, in the graph. This is done by minimizing

$$
\sum_{a, b}\left(\tilde{\boldsymbol{m}}^{a}-\tilde{\boldsymbol{m}}^{b}\right) W_{a b}
$$

with $\tilde{\boldsymbol{m}}^{a}$ the projection of $\boldsymbol{m}^{a}$ onto the low-dimensional manifold. Similarly to PCA, Eq. (6) can be reduced to a generalized eigenvector problem

$$
\mathcal{L} v=\lambda \boldsymbol{D} v
$$

Herein, $\boldsymbol{D}$ is the diagonal matrix of the graph with $D_{a a}=\sum_{b} W_{a b}$ and $\mathcal{L}$ the Laplacian of the graph given as $\mathcal{L}=\boldsymbol{D}-\boldsymbol{W}$. In the literature of LE, the eigenvalues are typically sorted in ascending order given the minimization of Eq. (6).

\subsection{Proposed metric}

In this work we propose a novel dissimilarity metric for groupwise registration based on LE,

$$
\mathcal{D}_{L E}=\Lambda_{1}
$$

Herein, $\Lambda_{1}$ is the second smallest eigenvalue from the generalized eigenvector problem defined in the previous subsection for LE, commonly known as the Fiedler eigenvalue or algebraic connectivity. ${ }^{13}$ The algebraic connectivity and its properties has been extensively studied. Its magnitude expresses the connectivity and robustness of the graph. An analog to PCA is made by Sharma et al. ${ }^{14}$ who state that the eigenvectors corresponding to the smallest eigenvalues can be interpreted as the axes of maximal variance of the graph. As such, a similar logic compared to PCA can be followed. More variance in the graph explained by the first eigenvalues (sorted in ascending order however) is associated with a better registration.

The eigendecomposition on an $s \times s$ matrix grows increasingly more time-consuming with respect to the number of samples $s$ under consideration. Therefore, we propose to use a stochastic gradient descent optimization method $^{15}$ to solve Eq. (1). With this method, a low number of samples (we use $s=2048$ ) is randomly selected in each iteration, which allows us to solve the generalized eigenvalue problem in an efficient manner. One should note that $\mathcal{L}$ is typically sparse (depending on the number of neighbours $k$ ), which also limits the computation time. For the optimization with the stochastic gradient descent method, the derivative of the cost function $\mathcal{C}$ with respect to the parameters $\boldsymbol{\mu}$ is required. To compute $\frac{\partial \mathcal{D}_{L E}}{\partial \boldsymbol{\mu}}$, we exploit the analytic expressions for derivatives of generalized eigenvalues given by de Leeuw. ${ }^{16}$

\section{EXPERIMENTS}

\subsection{Synthetic}

In a first experiment we show how LE is able to model nonlinear relationships in two synthetic images (40x30 pixels, Fig. 1(a)). We assess the evolution of the metric when these images are progressively translated with respect to each other and compare it to the metric evolution of PCA. The translations ranged from -5 voxels to +5 voxels with a step size of 0.1 voxels along the horizontal axis. 


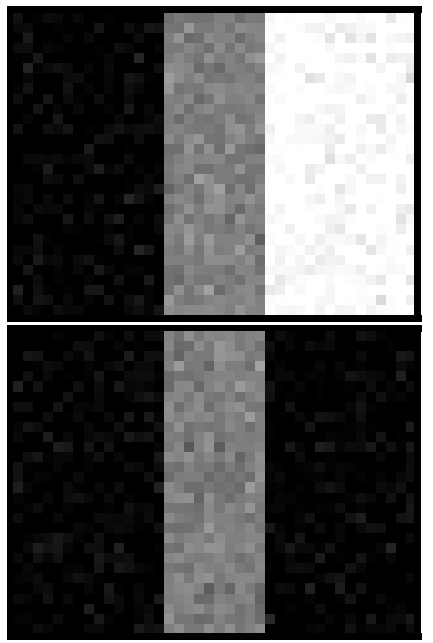

(a)

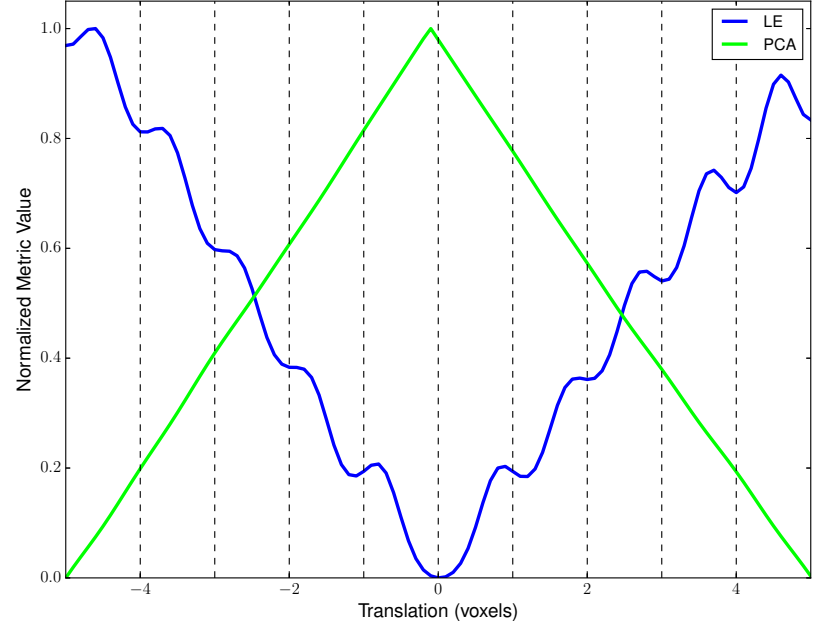

(b)

Figure 1: (a) Images used in the synthetic registration experiment. (b) Metric behaviour where the metric values were normalized with respect to their minimum and maximum.

\subsection{Carotid MR}

As a second experiment, registrations were performed on MRI acquisitions of the carotid artery under varying aqcuisition settings ${ }^{17}$ (Fig. 2). The experiment was performed to evaluate the added value of using a manifold instead of a hyperplane in quantitative MRI data. A total of 8 sequences was obtained with each containing 5 images under varying MR aqcuisition settings. A deformable transformation based on cubic B-splines was performed with a final grid spacing of $8 \mathrm{~mm}$. Registrations were performed with the proposed metric and are compared to the PCA-based metrics, ${ }^{5}$ average mutual information $(\mathrm{AMI})^{6}$ and with a conventional pairwise registration approach using mutual information (MI). All similarity metrics were regularized with a bending energy penalty for which the weights were determined empirically. The two free parameters in the LE-based metric, the number of nearest neighbours, $k$, and the diffusion time, $t$, were set to 100 and 0.5 respectively. The bifurcations of the carotid arteries were annotated as anatomical landmarks to quantify the registration accuracy with the groupwise target registration error (gTRE)

$$
g T R E(\boldsymbol{\mu})=\frac{1}{n} \sum_{i \neq r}^{n} \frac{1}{\left|P_{i}\right|} \sum_{j}^{\left|P_{i}\right|}\left\|\mathcal{T}_{i, r}\left(\boldsymbol{p}_{i, j}\right)-\boldsymbol{p}_{r, j}\right\| .
$$

\subsection{RIRE}

As a final experiment, registrations were performed on multimodal data of the brain to show the wide applicability of the proposed approach. We used the publicly publicly available RIRE database ${ }^{18}$ (Fig. 3), containing CT, PET and MRI acquisitions which were annotated with four to ten landmarks based on fiducial markers and a stereotactic frame. Rigid transformations were used to register the images and the LE-based metric was compared to PCA, PCA2, AMI and pairwise MI. The number of nearest neighbours, $k$, was set 100 and the diffusion time, $t$, to 0.25 for the LE-based metric. Registrations were considered to have failed following the criterion set by Tomaževič et al. ${ }^{19}$ These misregistrations were excluded from the analysis for each metric.

\section{RESULTS \& DISCUSSION}

In the registration performed on the synthetic images (Fig. 1(b)), it can be seen that the LE-based metric shows a number of local minima, corresponding to integer voxel displacement, and the correct global minimum achieved at zero displacement. The metric based on PCA shows an inverted behaviour, where a maximum is obtained 

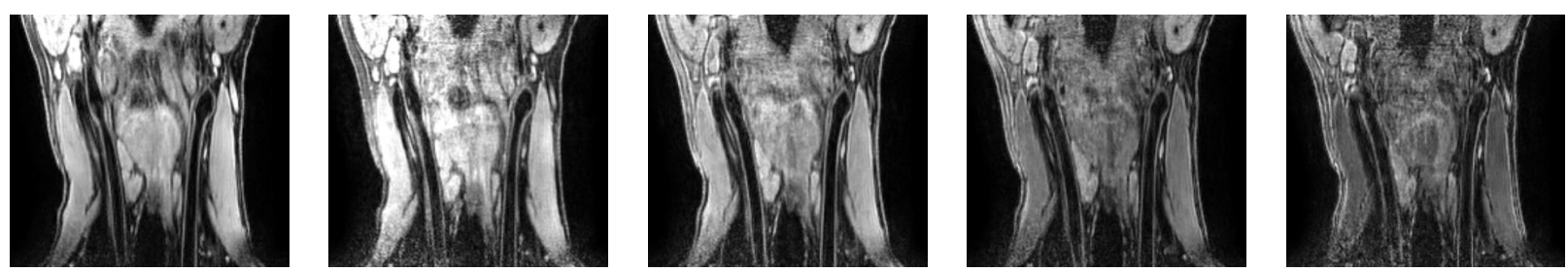

Figure 2: Image sequence used in the Carotid MR experiment

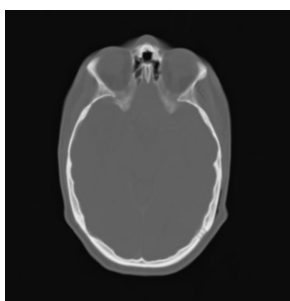

(a)

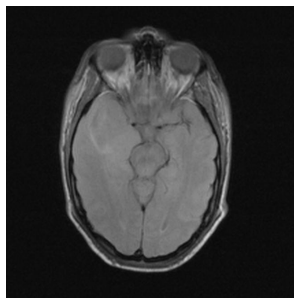

(b)



(c)

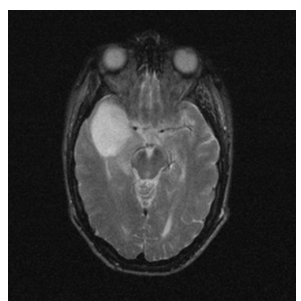

(d)



(e)

Figure 3: An example of the multimodal brain images of the RIRE database containing (a) CT, (b) MR-PD, (c) MR-T1, (d) MR-T2 and (e) PET images of the brain.

at zero displacement. A clear advantage in the metric behaviour of LE compared to PCA is found since no underlying linear model is present.

The results of the registration of the Carotid data are given in Table 1. The proposed LE metric performs best with a mean gTRE of $1.07 \pm 0.42 \mathrm{~mm}$ compared to PCA2 with $1.10 \pm 0.44 \mathrm{~mm}$ and AMI with $1.11 \pm 0.44 \mathrm{~mm}$. The improvement is non-significant compared to the other metrics. However, these results indicate that information carried by the nonlinear (as opposed to linear) dimensionality reduction technique can be beneficial for finding the optimal alignment. The proposed metric also performs better than pairwise MI with a mean gTRE of 1.10 \pm $0.42 \mathrm{~mm}$, implying the benefit of a groupwise approach in which all transformations are optimized simultaneously.

In Table 1 the results for the registration of the RIRE data can be found. The proposed methodology performs best amongst the groupwise approaches with a mean gTRE of $2.48 \pm 1.50 \mathrm{~mm}$ compared to $4.72 \pm 2.13 \mathrm{~mm}$ for AMI. PCA and PCA2 both misregister 16 patients, indicating their application is not suited for multimodal images with nonfunctional relationships. The LE-based metric significantly outperforms AMI at a significance of 0.05 ( $\mathrm{p}=0.02$, two-tailed Wilcoxon signed-rank test). This result indicates that even complex nonfunctional relationships can be modeled by the LE-based approach. For this experiment, pairwise MI obtains the best result, but does not significantly outperform the proposed method. We argue that setting the diffusion parameter to a single global parameter might not be beneficial in this dataset. Some sequences do not include a PET images, while some include only two MR images. Since the diffusion parameter is typically manifold-specific, ${ }^{7}$ a global setting for the diffusion might hinder the construction of a suitable manifold. In future work we plan to propose an automated parameter estimation for the diffusion.

Table 1: Results for, respectively, the registration of the Carotid and RIRE dataset. The values correspond to the groupwise target registration error (gTRE, in $\mathrm{mm}$ ) or the number of misregistrations.

\begin{tabular}{l|r} 
Method & Mean \pm Stdev \\
\hline MI & $1.10 \pm 0.41$ \\
\hline PCA & $1.15 \pm 0.45$ \\
PCA2 & $1.10 \pm 0.44$ \\
AMI & $1.11 \pm 0.44$ \\
LE & $1.07 \pm 0.42$
\end{tabular}

\begin{tabular}{l|rr} 
Method & Mean \pm Stdev & MisRegs \\
\hline MI & $2.48 \pm 0.82$ & 0 \\
\hline PCA & $4.94 \pm 4.08$ & 16 \\
PCA2 & $5.98 \pm 1.78$ & 16 \\
AMI & $4.72 \pm 2.13$ & 3 \\
LE & $2.68 \pm 1.50$ & 0
\end{tabular}




\section{CONCLUSION}

In this work we presented a novel dissimilarity metric for multimodal groupwise registration based on the nonlinear dimensionality reduction technique Laplacian eigenmaps. Results of three different experiments demonstrated increased accuracy and robustness compared to other state-of-the-art groupwise registration methodologies and competitive registration accuracy compared with pairwise MI, while carrying the benefits of groupwise registration.

\section{ACKNOWLEDGMENTS}

The research of Stefan Klein and Wiro J. Niessen is supported by the European Union Seventh Framework Programme (FP7/2007-2013) under grant agreement no. 601055, VPH-DARE@IT. Additionally, the authors would like to thank Dr. B.F. Coolen and Dr. A.J. Nederveen for providing the Carotid MR data.

\section{REFERENCES}

[1] Maes, F., Collignon, A., Vandermeulen, D., Marchal, G., and Suetens, P., "Multimodality image registration by maximization of mutual information," Medical Imaging, IEEE Transactions on 16(2), 187-198 (1997).

[2] Wells, W. M., Viola, P., Atsumi, H., Nakajima, S., and Kikinis, R., "Multi-modal volume registration by maximization of mutual information," Medical image analysis 1(1), 35-51 (1996).

[3] Spiclin, Z., Likar, B., and Pernus, F., "Groupwise registration of multimodal images by an efficient joint entropy minimization scheme," Image Processing, IEEE Transactions on 21(5), 2546-2558 (2012).

[4] Wachinger, C. and Navab, N., "Simultaneous registration of multiple images: Similarity metrics and efficient optimization," Pattern Analysis and Machine Intelligence, IEEE Transactions on 35(5), 1221-1233 (2013).

[5] Huizinga, W., Poot, D., Guyader, J.-M., Klaassen, R., Coolen, B., van Kranenburg, M., van Geuns, R., Uitterdijk, A., Polfliet, M., Vandemeulebroucke, J., Leemans, A., Niessen, W., and Klein, S., "PCA-based groupwise image registration for quantitative MRI," Medical Image Analysis 29, 65-78 (2016).

[6] Polfliet, M., Klein, S., Huizinga, W., de Mey, J., and Vandemeulebroucke, J., "The pythagorean averages as group images in efficient groupwise registration," in [2016 IEEE 13th International Symposium on Biomedical Imaging (ISBI)], 1261-1264, IEEE (2016).

[7] Belkin, M. and Niyogi, P., "Laplacian eigenmaps for dimensionality reduction and data representation," Neural computation 15(6), 1373-1396 (2003).

[8] Guerrero, R., Wolz, R., and Rueckert, D., "Laplacian eigenmaps manifold learning for landmark localization in brain mr images," in [International Conference on Medical Image Computing and Computer-Assisted Intervention], 566-573, Springer (2011).

[9] Wachinger, C. and Navab, N., "Entropy and laplacian images: Structural representations for multi-modal registration," Medical Image Analysis 16(1), 1-17 (2012).

[10] Azampour, M. F., Ghaffari, A., Hamidinekoo, A., and Fatemizadeh, E., "Manifold learning based registration algorithms applied to multimodal images," in [2014 36th Annual International Conference of the IEEE Engineering in Medicine and Biology Society], 1030-1034, IEEE (2014).

[11] Conjeti, S., Yigitsoy, M., Sheet, D., Chatterjee, J., Navab, N., and Katouzian, A., "Mutually coherent structural representation for image registration through joint manifold embedding and alignment," in [2015 IEEE 12th International Symposium on Biomedical Imaging (ISBI)], 601-604, IEEE (2015).

[12] Roweis, S. T. and Saul, L. K., "Nonlinear dimensionality reduction by locally linear embedding," Science 290(5500), 2323-2326 (2000).

[13] Fiedler, M., "Algebraic connectivity of graphs," Czechoslovak mathematical journal 23(2), 298-305 (1973).

[14] Sharma, A., Horaud, R. P., Knossow, D., and Von Lavante, E., "Mesh segmentation using Laplacian eigenvectors and gaussian mixtures.," in [AAAI Fall Symposium: Manifold Learning and Its Applications], (2009).

[15] Klein, S., Pluim, J. P., Staring, M., and Viergever, M. A., "Adaptive stochastic gradient descent optimisation for image registration," International journal of computer vision 81(3), 227-239 (2009).

[16] de Leeuw, J., "Derivatives of generalized eigen systems with applications," Department of Statistics, UCLA (2007). 
[17] Coolen, B. F., Poot, D. H., Liem, M. I., Smits, L. P., Gao, S., Kotek, G., Klein, S., and Nederveen, A. J., "Three-dimensional quantitative T1 and T2 mapping of the carotid artery: Sequence design and in vivo feasibility," Magnetic Resonance in Medicine (2015).

[18] West, J., Fitzpatrick, J. M., Wang, M. Y., Dawant, B. M., Maurer Jr, C. R., Kessler, R. M., Maciunas, R. J., Barillot, C., Lemoine, D., Collignon, A., et al., "Comparison and evaluation of retrospective intermodality brain image registration techniques," Journal of computer assisted tomography 21(4), 554-568 (1997).

[19] Tomaževič, D., Likar, B., and Pernuš, F., "Multi-feature mutual information image registration," Image Analysis $\mathcal{E}$ Stereology 31(1), 43-53 (2012). 